\title{
Entanglement of a Single Spin-1 Object: An Example of Ubiquitous Entanglement
}

\author{
Sinem Binicioğlu, ${ }^{1}$ M. Ali Can, ${ }^{2}$ Alexander A. Klyachko, ${ }^{3}$ \\ and Alexander S. Shumovsky ${ }^{1}$
}

Received November 13, 2006; revised January 28, 2007; Published online June 8, 2007

\begin{abstract}
Using a single spin-1 object as an example, we discuss a recent approach to quantum entanglement. [A.A. Klyachko and A.S. Shumovsky, J. Phys: Conf. Series 36, 87 (2006), E-print quant-ph/0512213]. The key idea of the approach consists in presetting of basic observables in the very definition of quantum system. Specification of basic observables defines the dynamic symmetry of the system. Entangled states of the system are then interpreted as states with maximal amount of uncertainty of all basic observables. The approach gives purely physical picture of entanglement. In particular, it separates principle physical properties of entanglement from inessential. Within the model example under consideration, we show relativity of entanglement with respect to dynamic symmetry and argue existence of single-particle entanglement. A number of physical examples are considered.
\end{abstract}

KEY WORDS: entanglement; quantum measurements; nonlocality.

PACS NUMBERS: 03.67 Mn; $03.65 \mathrm{Ud}$; 03.67-a.

\section{INTRODUCTION}

Recent development of quantum information technologies has led to a number of successful and promising realizations of protocols based on the use of quantum entanglement. For example, the quantum key distribution has recently become an industrial product. ${ }^{(1)}$ These developments caused

\footnotetext{
${ }^{1}$ Department of Physics, Bilkent University, Bilkent, Ankara 06800, Turkey; e-mail: sinem@fen.bilkent.edu.tr

2 Jet Propulsion Laboratory, California Institute of Technology, Pasadena, CA 91109-8099, USA.

${ }^{3}$ Department of Mathematics, Bilkent University, Bilkent, Ankara 06800, Turkey.
} 
a great burst of activity in investigation of quantum entanglement. During the last decade, the applications of entanglement to quantum information and quantum computing were discussed in a huge number of articles and review papers, in particular in Foundations of Physics. ${ }^{(2)}$

Nevertheless, the substance of entanglement still remains unclear, especially beyond the simplest case of two-qubit systems. Moreover, there is a certain muddle even in the very definition of entanglement because important and inessential are often jumbled together.

In this paper, our aim is not to discuss the applications but the physics behind the quantum entanglement.

For example, entanglement is usually associated with quantum nonlocality or violation of classical realism. ${ }^{(3-5)}$ This simply means that measurements on spatially separated parts of a quantum system may instantaneously influence one another. Physically this is caused by the quantum correlations between the parts of the system. ${ }^{(5)}$ Once created, those correlations keep on existing even after the spatial separation of parts.

On one hand, the nonlocality is probably the main distinguishing feature of quantum mechanics regarding classical physics. On the other hand, this notion does not contain any quantification of distance between separated entangled parts of a quantum system. Thus, it seems to be natural to assume that quantum system with strongly correlated intrinsic parts may manifest entanglement independent of distance between the parts and hence even as a local object without spatial separation of parts. ${ }^{(6-10)}$

The quantum nonlocality is often expressed in terms of violation of different Bell-type conditions of classical realism. ${ }^{(5)}$ This violation is a characteristic feature of entanglement in two-qubit systems. However, unentangled states of some systems beyond two qubits can also manifest the violation of those conditions. ${ }^{(11-13)}$ For example, the difference between entangled and unentangled states disappears for systems with dynamic symmetry group $\mathrm{SU}(\mathcal{H})$ with dimension of the Hilbert space $\operatorname{dim} \mathcal{H} \geq 3$ (see Ref. 14, cf. Ref. 15). As a matter of fact, violation of Bell-type conditions generally indicates the absence of "hidden" classical variables in quantum mechanics ${ }^{(5)}$ rather than entanglement (also see Appendix A).

This allows us to conclude that nonlocality and violation of classical realism alone are not the essential sign of entanglement and that there is no physical prohibition for the existence of entanglement of local objects (particles) caused by quantum correlations of their intrinsic degrees of freedom. ${ }^{(6-10)}$

Another common opinion is that the entanglement of multipartite systems defined in the Hilbert space $\mathcal{H}=\mathcal{H}_{A} \otimes \mathcal{H}_{B} \otimes \ldots$ can be 
associated with the nonseparability of states $\psi \in \mathcal{H}$ with respect to the parts of the system (e.g., see Ref. 16).

This statement, which is undoubtedly valid in the case of bipartite systems, does not have a lucid sense for multipartite entanglement. Stress that the notion of nonseparability is clearly useless in the case of singleparticle entanglement. $(9,10)$

Three-qubit states, whose classification has been constructed in Ref. 17 can be considered as an example. Namely, the states from the class, specified by the nonseparable GHZ (Greenberger-Horne-Zeilinger) state, manifest only three-partite entanglement with no correlations (entanglement) between pairs of qubits. In contrast, the nonseparable W-states manifest entanglement only between pairs of qubits while they are unentangled in the three-qubit sector. In turn, the so-called bi-separable states (separable with respect to one of the parties) may manifest bipartite entanglement. For details, see Appendix B.

Thus, it seems reasonable to set aside the criteria of entanglement based on nonlocality, violation of classical realism, and nonseparability, and to focus attention on physical manifestations of entanglement in the process of measurement of quantum observables.

Note first that there is a certain interdependence between quantum correlations peculiar to entangled states and quantum uncertainties (fluctuations) of local observables. ${ }^{(11,18,19)}$ Consider as an illustrative example the measurement of spin projection onto the quantization axis in the twoqubit states $\left|\psi_{\uparrow \uparrow}\right\rangle=|\uparrow \uparrow\rangle$ and $\left|\psi_{C E}\right\rangle=(|\uparrow \uparrow\rangle+|\downarrow \downarrow\rangle) / \sqrt{2}$. For the correlation functions and variances (uncertainties), we get

$$
\begin{aligned}
& \left\langle\psi_{\uparrow \uparrow}\left|\sigma_{z}^{A} ; \sigma_{z}^{B}\right| \psi_{\uparrow \uparrow}\right\rangle=0, \quad V\left(\sigma_{z}^{A, B} ; \psi_{\uparrow \uparrow}\right)=0, \\
& \left\langle\psi_{C E}\left|\sigma_{z}^{A} ; \sigma_{z}^{B}\right| \psi_{C E}\right\rangle=1, \quad V\left(\sigma_{z}^{A, B} ; \psi_{C E}\right)=1 .
\end{aligned}
$$

Here $\sigma_{z}^{A}, \sigma_{z}^{B}$ denote the $z$-component of Pauli spin operator,

$$
\left\langle\psi\left|\sigma^{A} ; \sigma^{B}\right| \psi\right\rangle=\left\langle\psi\left|\sigma^{A} \sigma^{B}\right| \psi\right\rangle-\left\langle\psi\left|\sigma^{A}\right| \psi\right\rangle\left\langle\psi\left|\sigma^{B}\right| \psi\right\rangle
$$

is the correlation function of local measurements, and

$$
V(\sigma ; \psi)=\left\langle\psi\left|\sigma^{2}\right| \psi\right\rangle-\langle\psi|\sigma| \psi\rangle^{2}
$$

is the variance of the observable $\sigma$ in the state $\psi$. Thus, the correlation functions and variances have similar behavior for the characteristic states like $\psi_{\uparrow \uparrow}$ and $\psi_{C E}$.

The natural question now is how many physical observables should be measured in order to conclude that a given state of a certain system is entangled. (20) This question has extremely high importance for 
understanding of physical essence of entanglement and its quantification. Besides that, this question has a quite practical meaning in connection with test of sources of entangled states. ${ }^{(21)}$

In a recent approach ${ }^{(7,11,22)}$ (for recent review, see Ref. 6), it has been proposed to begin the analysis of entanglement with the choice of independent basic observables that can be associated with the orthogonal basis of a certain Lie algebra $\mathcal{L}$. The corresponding Lie group $G=\exp (i \mathcal{L})$ defines the dynamic symmetry of the physical system under consideration.

It should be emphasized that the idea to specify a quantum system by accessible observables is known for a long time (e.g., see Ref. 23). Unfortunately, this principle idea is often set aside. As we show below in this paper, this principle plays extremely important role in description of quantum entanglement.

Within the approach of Refs. 7,11,22, the complete entanglement is interpreted as manifestation of quantum uncertainties of all basic observables at their extreme. By complete entanglement we mean here the maximal entanglement that can be achieved by pure states.

Note that, for a given quantum system, it is enough to know the completely entangled states because all other entangled states can be generated from those states through the use of SLOCC (stochastic local operations assisted by classical communications). ${ }^{(24,25)}$

The dual objective of present paper is to discuss the characteristic features of this approach, using a single qutrit (ternary quantum state) as an illustrative example of some considerable interest, and hence to justify existence of single-particle entanglement.

Qutrit is usually associated with ternary unit of quantum information. (26) Instructiveness of this example consists in the relativity of entanglement with respect to the choice of dynamic symmetry $G$ of ternary quantum physical system. Namely, one can choose either $G=S U(3)^{(27)}$ or $\mathrm{G}^{\prime}=\mathrm{SU}(2)$. Just the latter case of a single spin-1 system may manifest entanglement without division of the system into separated parts. $(7,9,10)$

Stress that entanglement of a single photon has been discussed for a long time. ${ }^{(28)}$ The picture always involves an external qubit formed by two possible paths owing to its propagation through a beam splitter. In contrast, our concept of the single-particle entanglement ${ }^{(6,10)}$ considers particle itself independent of its environment (see also important discussions in Refs. 9,8). In this case, quantum correlations peculiar to entanglement can be associated with intrinsic degrees of freedom of a particle.

In the paper, we show that the single-particle entanglement has all important properties of conventional two-qubit entanglement. In particular, its unentangled states are the spin-coherent states like those of two qubits $^{(11,12)}$ (concerning spin-coherent states, see the basic works ${ }^{(29)}$ and 
monographs $\left.{ }^{(30)}\right)$. In turn, the single-particle entangled states are squeezed like the two-qubit entangled states (for relation between squeezing and "conventional" multipartite entanglement, see Ref. 31).

Note that association of unentangled and coherent states on the one hand and entangled and squeezed states on the other hand blends well with the concept of entanglement as manifestation of quantum uncertainties at their extreme.

We also reveal the mechanism of intrinsic quantum correlations hidden behind the single-qutrit entanglement.

The paper is organized as follows. In Sec. 2, the principles of the dynamic symmetry approach to quantum entanglement are presented and we show existence of the single-particle entanglement for spin-1 systems. In Sec. 3, we discuss properties of that entanglement. Then, in Sec. 4, we consider some physical realizations. The conclusions are given in Sec. 5.

\section{ENTANGLEMENT AND QUANTUM FLUCTUATIONS}

As we have said in introduction, specifying a given quantum system, we should first choose the accessible independent physical observables associated with dynamic symmetry of the system. ${ }^{(6,11)}$

For example, in the case of a qubit (spin 1/2) system, dynamic symmetry is given by the group SU(2). The orthogonal basis of the corresponding Lie algebra $s u(2)$ consists of three spin operators (Pauli matrices). Thus, a two-qubit system is characterized by the dynamic symmetry $\mathrm{G}=\mathrm{SU}(2) \times \mathrm{SU}(2)$, which corresponds to the six basic observables (three Pauli matrices for each part). For the two-qubit pure state, the number of necessary measurements, providing information about entanglement carried by this state, is reduced to three. ${ }^{(20)}$ because of the local character of the measure of entanglement (concurrence) in this case. ${ }^{(32)}$

To illustrate special importance of the specification of quantum system by basic observables, consider a qutrit (ternary unit of quantum information) associated with a state

$$
|\psi\rangle=\sum_{s=-1}^{1} \psi_{s}|s\rangle, \quad \sum_{s=-1}^{1}\left|\psi_{s}\right|^{2}=1
$$

in the three-dimensional Hilbert space $\mathcal{H}_{3}$. As we have mentioned in introduction, there are at least two qualitatively different physical systems, whose states are qutrits. Namely, one possible realization corresponds to 
the general symmetry $\mathrm{G}=\mathrm{SU}(3)$ of the system, which implies eight basic observables (Gell-Mann matrices) ${ }^{(27)}$

$$
\begin{gathered}
\lambda_{1}=\left(\begin{array}{lll}
0 & 1 & 0 \\
1 & 0 & 0 \\
0 & 0 & 0
\end{array}\right), \quad \lambda_{2}=\left(\begin{array}{rrr}
0 & -i & 0 \\
i & 0 & 0 \\
0 & 0 & 0
\end{array}\right), \quad \lambda_{3}=\left(\begin{array}{rrr}
1 & 0 & 0 \\
0 & -1 & 0 \\
0 & 0 & 0
\end{array}\right), \\
\lambda_{4}=\left(\begin{array}{lll}
0 & 0 & 1 \\
0 & 0 & 0 \\
1 & 0 & 0
\end{array}\right), \quad \lambda_{5}=\left(\begin{array}{rrr}
0 & 0 & -i \\
0 & 0 & 0 \\
i & 0 & 0
\end{array}\right), \quad \lambda_{6}=\left(\begin{array}{lll}
0 & 0 & 0 \\
0 & 0 & 1 \\
0 & 1 & 0
\end{array}\right), \\
\lambda_{7}=\left(\begin{array}{rrr}
0 & 0 & 0 \\
0 & 0 & -i \\
0 & i & 0
\end{array}\right), \quad \lambda_{8}=\frac{1}{\sqrt{3}}\left(\begin{array}{rrr}
1 & 0 & 0 \\
0 & 1 & 0 \\
0 & 0 & -2
\end{array}\right) .
\end{gathered}
$$

Hereafter we call the corresponding system the true qutrit system.

Another realization assumes reduced symmetry $\mathrm{G}^{\prime}=\mathrm{SU}(2)$ of the physical system, which requires only three basic observables (spin-1 operators $)^{(10)}$

$$
\begin{gathered}
S_{x}=\frac{1}{\sqrt{2}}\left(\begin{array}{lll}
0 & 1 & 0 \\
1 & 0 & 1 \\
0 & 1 & 0
\end{array}\right), \quad S_{y}=\frac{1}{\sqrt{2}}\left(\begin{array}{rrr}
0 & -i & 0 \\
i & 0 & -i \\
0 & i & 0
\end{array}\right), \\
S_{z}=\left(\begin{array}{rrr}
1 & 0 & 0 \\
0 & 0 & 0 \\
0 & 0 & -1
\end{array}\right) .
\end{gathered}
$$

We call this case the spin-qutrit system.

As we discuss in the next section, qutrit (1) may manifest entanglement in the case of single spin-qutrit system, while single true qutrit can never be entangled.

Before we begin to consider entanglement of a single qutrit, let us briefly discuss the physical definition of entanglement of Refs. 7,11,22.

For a given state $\psi$ of a system with basic observables $X_{i}$, we can measure the expectation values $\left\langle\psi\left|X_{i}\right| \psi\right\rangle$ and variances (uncertainties)

$$
V\left(X_{i} ; \psi\right)=\left\langle\psi\left|X_{i}^{2}\right| \psi\right\rangle-\left\langle\psi\left|X_{i}\right| \psi\right\rangle^{2} .
$$

It is interesting that Wigner and Yanase ${ }^{(33)}$ have proposed a new quantity to measure specific quantum information about a state $\psi$, that 
can be obtained from macroscopic measurement of the observable $X_{i}$ in this state (the so called Wigner-Yanase skew information):

$$
I_{W Y}\left(X_{i} ; \rho\right)=-\frac{1}{2} \operatorname{Tr}\left(\left[\sqrt{\rho}, X_{i}\right]^{2}\right) .
$$

In the case of pure state $\psi$, the density matrix takes the form $\rho=|\psi\rangle\langle\psi|$, the quantity (5) simply coincides with the variance (4) (see Refs. 34 for further discussion of Wigner-Yanase quantum "skew information").

Following ${ }^{(11,7,22)}$ introduce the total variance

$$
\mathbb{V}(\psi)=\sum_{i} V\left(X_{i} ; \psi\right)
$$

calculated for all basic observables and all parts of the system (in the case of multipartite systems). By definition, this quantity (6) is an invariant, independent of the choice of basis of the Lie algebra $\mathcal{L}$ of observables. This quantity (6) can also be interpreted as the total amount of WignerYanase information peculiar to the state $\psi$.

It was proposed in Refs. 7,11,22 that, complete entangled states $\psi_{C E}$ of an arbitrary system can be defined in terms of maximum of total variance:

$$
\mathbb{V}\left(\psi_{C E}\right)=\max _{\psi \in \mathcal{H}} \mathbb{V}(\psi)
$$

This definition has a simple physical meaning. It associates complete entanglement with the maximal amount of quantum uncertainty in a given system. Validity of this definition in some known cases of completely entangled states of multipartite systems has been shown in a number of papers (see Ref. 6 for references).

It is seen that Eq. (7) represents a certain variational principle, similar in a sense to the maximal entropy principle in statistical physics, which is used to define equilibrium states.

At first glance, Eq. (7) defines only completely entangled states $\psi_{C E}$. In fact, it can be used to specify all entangled pure states of the system as well. The point is that all entangled states of a given system are equivalent to SLOCC. ${ }^{(24,25)}$ Note that SLOCC are represented by operators from the complexified dynamic symmetry group ${ }^{(25)}$

$$
\widehat{S O C C} \equiv g^{c} \in G^{c}=\exp (\mathcal{L} \otimes \mathbb{C}) .
$$

Thus, for the entangled states $\psi_{E}$ we get

$$
\left|\psi_{E}\right\rangle=g^{c}\left|\Psi_{C E}\right\rangle
$$


Note that in the case of compact Lie algebra (like $\mathrm{SU}(N)$ ), the quadratic form

$$
\sum_{i} X_{i}^{2}=C_{\mathcal{H}}
$$

is a scalar (Casimir operator). Then Eq. (6) takes the form

$$
\mathbb{V}(\psi)=C_{\mathcal{H}}-\sum_{i}\left\langle\psi\left|X_{i}\right| \psi\right\rangle^{2}
$$

It is easily seen that the maximum of the total variance (9) is provided by the condition

$$
\forall i \quad\left\langle\psi_{C E}\left|X_{i}\right| \psi_{C E}\right\rangle=0
$$

This condition represents a set of algebraic equations for the complex coefficients of the wave function $|\psi\rangle$, which enables us to fairly simplify the analysis of entanglement. Validity of this condition (10) for completely entangled qubit-states in quite general settings has been checked in Ref. 18. Because the condition (10) deals directly with measurement of physical observables, it has been proposed in Ref. 18 to use the condition as an operational definition of complete entanglement.

Amount of entanglement carried by entangled states (8) can also be measured by means of total variance as follows ${ }^{(35)}$

$$
\mu(\psi)=\sqrt{\frac{\mathbb{V}(\psi)-\mathbb{V}_{\min }}{\mathbb{V}_{\max }-\mathbb{V}_{\min }}} .
$$

Here $\mathbb{V}_{\max }$ and $\mathbb{V}_{\min }$ denote the total variance for completely entangled and unentangled states, respectively. This measure coincides with the concurrence $^{(36)}$ for pure states of an arbitrary bipartite system. It can also be applied beyond bipartite systems. For unentangled states, $\mu(\psi)=0$, while for entangled states it lies in $(0,1]$, so that $\mu\left(\psi_{C E}\right)=1$.

\section{ENTANGLEMENT IN A SINGLE SPIN-QUTRIT SYSTEM}

Note that the definition of complete entanglement (7) and its equivalent form (10) do not assume the multipartite character of quantum systems. 
Does the single qubit obey the condition (10)? The answer is not. The point is that the pure single-qubit state

$$
\psi=a|\uparrow\rangle+b|\downarrow\rangle, \quad|a|^{2}+|b|^{2}=1
$$

is in fact characterized by only two real parameters $(|a|$ and $\arg a-\arg b)$, for which three Eq. (10) with Pauli matrices as basic observables have only trivial solution.

For decades, qubits remain the main object of quantum information. Therefore, nonexistence of single-qubit entanglement is frequently used as a general argument against the single-particle entanglement (see nice discussion in Ref. 8).

We now turn to the qutrit (1), which is specified by five real parameters. Equation (10) with eight basic observables (2) clearly have only trivial solutions, so that single true qutrit system does not manifest entanglement like single qubit system.

Situation changes qualitatively if qutrit (1) is considered as a state of spin-qutrit system with only three basic observables (3). ${ }^{(10)}$ In this case, Eq (10) with three spin-1 operators (3) have nontrivial solutions, so that complete entanglement of a single spin qutrit system is allowed. In particular, it is straightforward to calculate the measure (11) for the single spin-qutrit state:

$$
\mu(\psi)=2\left|\psi_{-1} \psi_{1}-\psi_{0}^{2} / 2\right|
$$

Thus, the state (1) of a single spin-1 system manifests entanglement if its coefficients obey the condition

$$
\frac{1}{4} \geq\left|\psi_{-1}\right|^{2}\left|\psi_{1}\right|^{2}+\frac{1}{4}\left|\psi_{0}\right|^{2}-\left|\psi_{-1}\right|\left|\psi_{0}\right|\left|\psi_{1}\right|^{2} \cos \left(\phi_{-1}+\phi_{1}-2 \phi_{0}\right)>0 .
$$

Here $\phi_{\ell}=\arg \psi_{\ell}$. Complete entanglement is achieved when this form (13) takes the value $1 / 4$. For example, the states

$$
\left|\psi_{0}\right\rangle=|0\rangle
$$

and

$$
\left|\psi_{ \pm}\right\rangle=\frac{1}{\sqrt{2}}(|1\rangle \pm|-1\rangle)
$$

are completely entangled qutrit states of a single spin-qutrit system. 
Before we begin to discuss the precise meaning of the above obtained result, let us stress the relativity of entanglement with respect to dynamic symmetry of physical system. The same state (1) is unentangled if dynamic symmetry of the system is $\mathrm{G}=\mathrm{SU}(3)$ and entangled in the case of reduced dynamic symmetry $\mathrm{G}^{\prime}=\mathrm{SU}(2)$.

To interpret entanglement of single spin-qutrit system, let us compare it with two-qubit entanglement that has been scrutinized thoroughly.

It is known that an entangled two-qubit state is associated with the SU(2) squeezed states, ${ }^{(31)}$ while unentangled states are the SU(2) coherent states. ${ }^{(11,12)}$ We now show that this interpretation is valid for the entangled and unentangled states of a single spin-qutrit as well.

Let us begin with the SU(2) coherent states that, for a spin $s$, are defined in the following way $(29,30)$

$$
|\alpha\rangle=D(\alpha)|-s\rangle, \quad \alpha \in \mathbb{C},
$$

where

$$
D_{\alpha}=\exp \left(\alpha S_{+}-\alpha^{*} S_{-}\right)
$$

and $|-s\rangle$ is the lowest state among the $(2 s+1)$ states of spin- $s$ system. Here

$$
S_{ \pm}=S_{x} \pm i S_{y}
$$

are the spin rising and lowering operators, respectively.

In the "vacuum" state $|-s\rangle$, the spin has a given projection $-s$ onto the $z$-axis $\left\langle-s\left|S_{z}\right|-s\right\rangle=-s$, so that the corresponding variance $V\left(S_{z} ;-s\right)=0$. For the two other spin operators in the direction orthogonal to the quantization axis $z$ we get

$$
\begin{array}{r}
\left\langle-s\left|S_{x}\right|-s\right\rangle=\left\langle-s\left|S_{y}\right|-s\right\rangle=0, \\
V\left(S_{x} ;-s\right)=V\left(S_{y} ;-s\right)=s / 2,
\end{array}
$$

so that the total variance (6) takes the from

$$
\mathbb{V}(-s)=s
$$

This is the minimal value of the total variance for the spin-s system under consideration. Thus, in view of the definition of entanglement, given in the previous section, the state $|-s\rangle$ is unentangled. 
According to Eq. (9), the maximum of the total variance of a single spin- $s$ system is

$$
\mathbb{V}_{\text {max }}=\mathbb{V}\left(\psi_{C E}\right)=s(s+1)
$$

This allows us to represent the measure of entanglement (11) for a single spin-s system in the following form

$$
\mu(\psi)=\frac{1}{s} \sqrt{\mathbb{V}(\psi)-s}
$$

Thus, the measure (11) vanishes for coherent states.

It is easily seen that, in the case of a single qubit $(s=1 / 2)$, any state of the system is a coherent one. While in the case of single spin-qutrit ( $s=$ 1), coherent states (16) have the form

$$
\begin{aligned}
|\alpha\rangle= & \frac{e^{2 i \phi}}{2}[1-\cos (2|\alpha|)]|+1\rangle+\frac{e^{i \phi}}{\sqrt{2}} \sin (2|\alpha|)|0\rangle \\
& +\frac{1}{2}[1+\cos (2|\alpha|)]|-1\rangle,
\end{aligned}
$$

where $\phi=\arg \alpha$. Substituting coefficients of the state (19) into Eq. (12), we can see that the measure of entanglement vanishes like in the case of state $|-1\rangle$. This is natural. The point is that the operator $\left(\alpha S_{+}-\alpha^{*} S_{-}\right)$in (17) belongs to the $s u(2)$ algebra, so that the displacement operator (17) amounts to an $\mathrm{SU}(2)$ rotation. This means that every spin coherent state (16) is just a state with minimal spin projection $-s$ onto some direction, which can be chosen as a new quantization axis. Thus, there is no principle difference between the spin coherent state and state $|-s\rangle$. In particular, spin coherent state is as unentangled as the state $|-s\rangle$.

Consider now the spin squeezed state for the spin-qutrit system under consideration.

Following Kitagawa and Ueda, ${ }^{(37)}$ we call spin state $\xi$ to be squeezed iff $V_{r}(\xi)<s / 2$ for some direction $r \perp \vec{s}$, where

$$
\vec{s}=\vec{e}_{x}\left\langle S_{x}\right\rangle+\vec{e}_{y}\left\langle S_{y}\right\rangle+\vec{e}_{z}\left\langle S_{z}\right\rangle
$$

is the direction of the average spin vector.

This means that in a coordinate system with the $z$-axis along the average spin vector $\vec{s}$, we always have

$$
V_{x}(\xi)+V_{y}(\xi) \geq s
$$


in contrast to the spin-coherent state. It is easy to check that this condition of squeezing (20) is valid for the states (14) and (15), therefore they are squeezed.

Some spin-squeezed states can be constructed by means of the squeezing operator ${ }^{(37)}$

$$
\mathcal{S}(\xi)=\exp \left[\left(\xi^{*} S_{-}^{2}-\xi S_{+}^{2}\right) / 2\right]
$$

so that

$$
|\xi\rangle=\mathcal{S}(\xi)|-1\rangle=-e^{i \varphi} \sin |\xi||1\rangle+\cos |\xi||-1\rangle .
$$

Here $\varphi=\arg \xi$. The measure (11) for this state is

$$
\mu(\xi)=|\sin (2|\xi|)|
$$

Thus, this state is entangled if $|\xi| \neq k \pi / 2, k=0,1, \ldots$. At $\xi=\pi / 4+k \pi$, this state coincides with (15) and hence manifests complete entanglement.

Note that in the case of a single qubit, the squeezing operator is simply the identity operator. The squeezed states of two qubits are usually associated with a sort of "two-mode" squeezing ${ }^{(31)}$

Thus, for the single spin-qutrit system, coherent states are unentangled while squeezed states manifest entanglement like in the case of conventional two-qubit states. Stress that this correspondence stays within the framework of the definition of entanglement based on Eqs. (7) and (8).

At the very beginning of the paper, we have stated that the singleparticle entanglement is caused by quantum correlations between intrinsic degrees of freedom of the particle. The general picture of those correlations can be revealed through the use of well known formal correspondence between the states of single spin-qutrit and two qubits, in other words, of two spin- $1 / 2$ and single spin- 1 . This correspondence is given by the Clebsch-Gordon decomposition:

$$
\mathcal{H}_{2} \otimes \mathcal{H}_{2}=\mathcal{H}_{3} \oplus \mathcal{H}_{A}
$$

Here $\mathcal{H}_{2}$ denotes the two-dimensional Hilbert space of states of a single spin- $\frac{1}{2}, \mathcal{H}_{3}$ is the three-dimensional Hilbert space of spin-1, corresponding to the symmetric triplet of states in the basis of $\mathcal{H}_{2} \otimes \mathcal{H}_{2}$, while $\mathcal{H}_{A}$ corresponds to the antisymmetric singlet in the basis of $\mathcal{H}_{2} \otimes \mathcal{H}_{2}$. Denoting the 
basis in $\mathcal{H}_{2}$ by $|\uparrow\rangle$ and $|\downarrow\rangle$, we obtain the basis in $\mathcal{H}_{3}$ in the following form

$$
|s\rangle= \begin{cases}|\uparrow \uparrow\rangle, & \text { projection of total spin } s=1 \\ \left.\frac{1}{\sqrt{2}}(|\uparrow \downarrow\rangle+\downarrow \uparrow\rangle\right), & \text { projection of total spin } s=0 \\ |\downarrow \downarrow\rangle, & \text { projection of total spin } s=-1\end{cases}
$$

while the antisymmetric singlet is

$$
|A\rangle=\frac{1}{\sqrt{2}}(|\uparrow \downarrow\rangle-|\downarrow \uparrow\rangle) .
$$

If we now assume that the singlet state (23) is forbidden because of some physical reasons, then the system of two qubits becomes exactly equivalent to the spin-qutrit system. Note, that one of the symmetric states in (22) is completely entangled in the two-qubit sector. This state is clearly equivalent to the state (14), which is completely entangled in the spinqutrit sector as well. On making the further assumption that spin-qutrit is a local object (particle), we have to associate the two qubits with intrinsic degrees of freedom of this object. Simply there is no alternative.

Thus, the single spin-qutrit entanglement can be interpreted in terms of quantum correlations between the two intrinsic qubits under the following conditions:

1. The Hilbert space of two qubits does not contain antisymmetric states.

2. System of two qubits is a local one, so that we can neglect the spatial separation of the qubits and thus interpret them as intrinsic degrees of freedom of a single "particle".

In the next section, we turn to the discussion of possible physical realizations of spin-qutrit entanglement.

\section{PHYSICAL REALIZATIONS OF SINGLE SPIN-QUTRIT ENTANGLEMENT}

Qubit systems are often associated with two-level atoms, where quantum correlations between the atoms can be generated either by photon exchange or by means of dipole-dipole interaction. In the latter case, decrease of the interatomic distance down to the lamb-Dicke limit (interatomic separation becomes much shorter than the wavelength of two-level transition) leads to an effective discard of the antisymmetric 


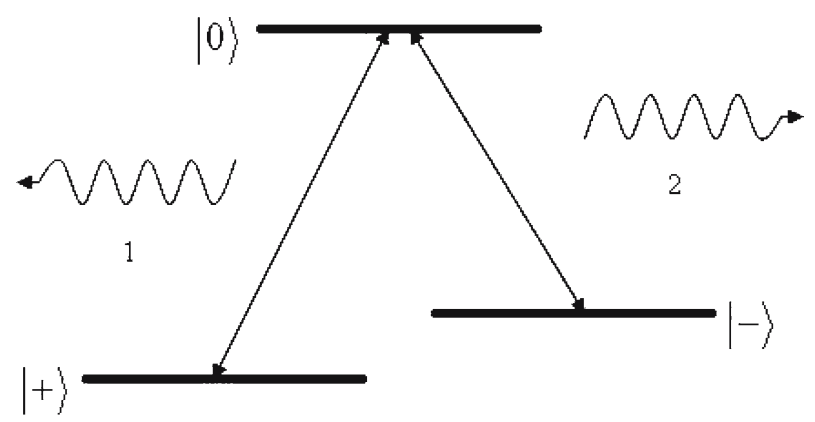

Fig. 1. Interaction between $\lambda$-type three-level atom and two cavity modes.

state. $^{(38)}$ Thus, this two-qubit system behaves like a single spin-qutrit object.

The nice feature of this example is that the reduction of symmetry

$$
\left.[\mathrm{SU}(2) \times \mathrm{SU}(2)]_{(\text {in }} 4 \text { dimensions }\right) \rightarrow \mathrm{SU}(2)(\text { in } 3 \text { dimensions })
$$

and localization accompany each other.

Another example is provided by the so-called biphoton, i.e. by two photons created at once and propagating along the same direction (see Ref. 39 and references therein). With respect to polarization, this object represents the $\mathrm{SU}(2)$ ternary system (spin-qutrit) and is as local as a single photon. Antisymmetric state with respect to permutations is forbidden here by the Bosonic nature of photons. Undoubtedly, biphoton can be split into spatially separated photons, carrying polarization qubits. But before splitting, it should be considered as a local spin-qutrit object.

Apologists of the standpoint that entanglement is inherent to systems with spatially separated parties can say that the above two examples do not fit the notion of a single particle. Therefore, we now turn to examples that definitely correspond to a single particle entanglement.

An important example of the SU(2) ternary system is provided by the three-level atom with $\lambda$-type transition shown in Fig. 1.

Here the highest excited level can be associated with the state $|0\rangle$ of "spin" 1 , while the two lower levels with the states $|+1\rangle$ and $|-1\rangle$, respectively.

The Hamiltonian, describing interaction between the atom and two cavity modes, has the form

$$
H_{\text {int }}=g_{1} R_{0+} a_{1}+g_{2} R_{0-} a_{2}+H . c .,
$$


where $g_{i}$ denotes the corresponding coupling constant, $R_{b c}=|b\rangle\langle c|$ is the atomic operator, and $a_{i}$ is the photon annihilation operator for the field mode $i=1,2$. The spin operators (3) have the form

$$
\begin{aligned}
S_{x} & =\frac{1}{\sqrt{2}}\left(R_{+0}+R_{0+}+R_{0-}+R_{-0}\right), \\
S_{y} & =\frac{-i}{\sqrt{2}}\left(R_{+0}+-R_{0+}+R_{0-}-R_{-0}\right), \\
S_{z} & =R_{++}-R_{--} .
\end{aligned}
$$

In view of the results of Sec. 3, the state $\left|\psi_{i n}\right\rangle=|0\rangle \otimes|\mathrm{vac}\rangle$ of the atom-field system, in which the atom is in excited state and cavity field is in the vacuum state, is completely entangled with respect to the atomic observables given by Eq. (25). Under influence of the atom-photon interaction (24), this state passes to the following normalized state

$$
\frac{1}{\sqrt{g_{1}^{2}+g_{2}^{2}}}\left(g_{1}|+\rangle \otimes\left|1_{1}\right\rangle+g_{2}|-\rangle \otimes\left|1_{2}\right\rangle\right)
$$

and vice versa. This state (26) can be interpreted as the two-qubit state, where one qubit is formed by the atomic states $| \pm\rangle$ and the second qubit by the photon states $\left|1_{1}\right\rangle$ and $\left|1_{2}\right\rangle$. Clearly, this state is entangled, and the corresponding concurrence ${ }^{(36)}$ has the form

$$
\mu=\frac{2\left|g_{1} g_{2}\right|}{g_{1}^{2}+g_{2}^{2}} .
$$

This example clearly illustrates decay of the single spin-qutrit entangled state $\left|\psi_{i n}\right\rangle$ into the two-qubit entanglement. The atom and photon qubits can be spatially separated by cavity leakage.

Another important example of the SU(2) entanglement of single particle is provided by the isotriplet of $\pi$-mesons. For detailed discussion of this example we refer recent work. ${ }^{(6)}$

The above example of meson isotriplet is similar to the Cooper pairs in superfluid phases of ${ }^{3} \mathrm{He}$. It is well known that the atoms of ${ }^{3} \mathrm{He}$ have spin $s=\frac{1}{2}$ each and that the total spin of a Cooper pair is $s=1$, so that the antisymmetric state of two atomic qubits is forbidden. ${ }^{(40)}$ Note that in the BCS superconductors where $s=0$, the only allowed pair wave function is given by the antisymmetric singlet state (23).

Another simple example of a single particle with spin 1, which can manifest entanglement, is provided by the deuteron, which is a nucleus of 
a deuterium atom, consisting of weakly bounded proton and neutron. ${ }^{(41)}$ Note that, unlike $\pi^{0}$ meson, this is a stable particle. Each nucleon in the deuteron can be considered as an intrinsic qubit with respect to its spin $\frac{1}{2}$. An experimental proof of the existence of entanglement in deuteron and of the possible use of it for quantum teleportation of spin states of massive particles has been reported recently Ref. 42 .

It is possible to find many other examples, from the spin-1 atoms like ${ }^{87} \mathrm{Rb}$ and ${ }^{23} \mathrm{Na}$, widely used in investigation of Bose-Einstein condensation, to the more exotic systems like vector mesons and three spin-1 gauge bosons in the standard model ${ }^{(43)}$ in which spin-qutrit entanglement may be realized.

\section{CONCLUSION}

We have argued existence of single spin-qutrit entanglement. The instructive significance of this system is that it allows twofold consideration as a single spin-1 object and as two qubits, defined in the symmetric sector of the Hilbert space. This correspondence allows us to interpret entanglement of single spin-qutrit as manifestation of quantum correlations between the intrinsic qubit degrees of freedom. We have shown that entanglement of single spin-qutrit particle may take place independent of whether or not the intrinsic qubits can be separated. Thus, the single spin-qutrit entanglement does not fit conventional requirements of nonseparability and nonlocality. At the same time, the single spin-qutrit entanglement has all physical features of two-qubit entanglement. In particular, entangled states of a single spin-qutrit are squeezed and unentangled states are coherent like in the case of bipartite systems.

We have discussed a number of physical objects that can be prepared in entangled spin-qutrit states. We have shown that the physical condition of complete entanglement as extreme of quantum fluctuations can be important for understanding of low stability of entangled states of particles.

The obtained result about the single-particle entanglement for the spin-qubit system is clearly valid for all systems with high enough dynamic symmetry $\mathrm{SU}(N)$ at $N \geq 3$.

The obtained results show distinctly that the physical definition of entanglement ${ }^{(6)}$ based on definition of basic observables and their quantum fluctuations, is more general than the previous definitions that appeal to nonlocality and nonseparability. As we have shown, using true and spin qutrits as an illustrative example, the presetting of basic observables plays crucial role in the description of entanglement. In particular, it defines 
specific relativity of entanglement with respect to dynamic symmetry of physical system. The definition in terms of the variational principle (7) can be used for investigation of entanglement of different physical objects, including elementary particle, quasi-particle excitations in condensed matter and so on. Thus, it essentially broaden the applicability of this notion beyond the bounds of quantum information. It is possible to say that the association of entanglement with quantum uncertainties of basic observables makes this notion to be ubiquitous in physics.

The possibility of experimental observation of single-particle entangled states represents a problem of high importance and deserves special discussion. Let us only note that the decay of a single entangled SU(2) qutrit into two entangled qubits may be used for this aim.

In our analysis, we have used a general approach to quantum entanglement, ${ }^{(6)}$ which assigns the primary importance to the dynamic symmetry properties of physical systems.

We have restricted our consideration by pure states. In the future, we hope to extend our approach on the mixed states. In particular case of single SU(2) qutrit, the mixed state entanglement can be quantified in the same way as the two-qubit entanglement (see Appendix C). So far, the measure of mixed-state entanglement is known only for two qubits (see the first reference in Ref. 36). The principle difficulty here is that the total variance of mixed states contains contributions of both quantum and classical (statistical) uncertainties. The problem of detachment of the two principally different contributions deserves special discussion. The ideas related to the Wigner-Yanase quantum information ${ }^{(33,34)}$ may be useful here.

\section{ACKNOWLEDGMENTS}

Part of this work was carried out (by M.A.C.) at the Jet Propulsion Laboratory, California Institute of Technology, under a contract with the National Aeronautics and Space Administration (NASA). M.A.C. also acknowledges support from the Oak Ridge Associated Universities (ORAU) and NASA.

\section{APPENDIX A: CLASSICAL REALISM}

The problem of classical realism can be formulated in the following way. It is known that the measurement of a quantum mechanical observable $X_{i}$ in a given state $\psi$ produces random quantity $x_{i}$ determined by expectations $\left\langle\psi\left|f\left(X_{i}\right)\right| \psi\right\rangle$ of all functions $f\left(x_{i}\right)$ (it is usually enough to 
consider only moments $f\left(x_{i}\right)=x_{i}^{n}$ (see Ref. 44). In the case of commuting quantum observables $\left[X_{i}, X_{j}\right]=0$, the corresponding random quantities $x_{i}$ and $x_{j}$ have the same joint probability distribution. Einstein's idea of classical realism. ${ }^{(3)}$ assumes that all quantum observables have the same hidden joint distribution independent of whether they are commuting or not.

Bell's approach to prove nonexistence of hidden variables is based on formulation of certain "classical" conditions on measurement of quantum observables and check of their violation in quantum mechanics. ${ }^{(5)}$ Note that, from the mathematical point of view, problem of Bell's conditions lies within the problem of distributions with given margins $(11,13)$ and that practically all known and many still unknown Bell-type inequalities were obtained in mathematics even before the formulation of problem by Bell.

It has been proved in Ref. 11 that an irreducible quantum system with dynamic symmetry group $G$ of $\operatorname{rank} \operatorname{rk}(G) \geq 2$ is incompatible with classical realism, i.e. violates Bell-type conditions. Since $\operatorname{rk}(\operatorname{SU}(n))=n-1$, state of a single true qutrit with the dynamic symmetry group $G=\mathrm{SU}$ (3) violate Bell's conditions of classical realism. It has been shown in Sec. 3 that the true qutrit states do not manifest entanglement.

\section{APPENDIX B: THREE-QUBIT ENTANGLEMENT}

Three-qubit states may manifest entanglement of two different types. Namely, entanglement caused by correlations of all three qubits and entanglement due to correlation between pair of parts. ${ }^{(17)}$ The three-qubit entanglement is measured by means of 3 -tangle, ${ }^{(17,45)}$ which for the general state

$$
|\psi\rangle=\sum_{k, \ell, m=0}^{1} \psi_{k \ell m}|k \ell m\rangle, \quad \sum_{k, \ell, m=0}^{1}\left|\psi_{k \ell m}\right|^{2}=1,
$$

has the form

$$
\begin{aligned}
\tau(\psi)= & 4 \mid \psi_{000}^{2} \psi_{111}^{2}+\psi_{001}^{2} \psi_{110}^{2}+\psi_{010}^{2} \psi_{101}^{2}+\psi_{100}^{2} \psi_{011}^{2} \\
& -2\left(\psi_{000} \psi_{001} \psi_{110} \psi_{111}+\psi_{000} \psi_{010} \psi_{101} \psi_{111}+\psi_{000} \psi_{100} \psi_{011} \psi_{111}\right. \\
& \left.+\psi_{001} \psi_{010} \psi_{101} \psi_{110}+\psi_{001} \psi_{100} \psi_{011} \psi_{110}+\psi_{010} \psi_{100} \psi_{011} \psi_{101}\right) \\
& +4\left(\psi_{000} \psi_{011} \psi_{101} \psi_{110}+\psi_{001} \psi_{010} \psi_{100} \psi_{111}\right) \mid
\end{aligned}
$$


According to classification by Miyake, ${ }^{(17)}$ the following three states

$$
\begin{aligned}
|G H Z\rangle & =\frac{1}{\sqrt{2}}(|000\rangle+|111\rangle), \\
|W\rangle & =\frac{1}{\sqrt{3}}(|011\rangle+|101\rangle+|110\rangle), \\
|B i\rangle & =\frac{1}{\sqrt{2}}(|011\rangle+|101\rangle)
\end{aligned}
$$

are generic for the three SLOCC-nonequivalent classes in the eight-dimensional Hilbert space. The non-separable states from the GHZ class manifest only three-partite entanglement (3-tangle (B.1) has nonzero values for the states from this class), while any pair of qubits is unentangled. The latter can be checked by reduction of the three-qubit density matrix $\rho_{G H Z}=$ $|G H Z\rangle\langle G H Z|$ to the two-qubit mixed state $\rho_{G H Z}^{\prime}=\operatorname{Tr}_{\text {single }} \rho_{G H Z}$, where $\operatorname{Tr}_{\text {single }}$ denotes trace over one of the parts, with the subsequent calculation of the concurrence, which in this case always have zero value.

In turn, the non-separable states from the $\mathrm{W}$ class always have zero 3-tangle and hence do not manifest three-partite entanglement. In turn, any bipartite reduced state with the density matrix $\rho^{\prime}{ }_{W}=\operatorname{Tr}(|W\rangle\langle W|)$ has nonzero concurrence and therefore shows bipartite entanglement.

Finally, the separable states from the Bi class are similar, in a sense, to the W states. Namely, they always have zero 3-tangle while manifest bipartite entanglement (for two given qubits only).

Thus, the nonseparability (separability) of the three-qubit states does not indicate identically the presence (absence) of entanglement and its type in contrast to the bipartite systems.

In the latter case, entanglement is usually associated with specific behavior of entropy of the reduced state single-part state. Namely, entanglement of two qubits with statistical state $\rho_{A B}$ exists if $H\left(\rho_{A}\right)=H\left(\rho_{B}\right) \neq$ 0 , where $H(\rho)=-\operatorname{Tr}(\rho \log \rho)$ is the von Neumann entropy. The maximal (complete) entanglement corresponds to the reduced states that have the diagonal form

$$
\rho_{r}=\left(\begin{array}{cc}
1 / 2 & 0 \\
0 & 1 / 2
\end{array}\right), \quad r=A, B,
$$

in a certain basis of the two-dimensional single-qubits space. ${ }^{(46)}$

In the above case of three-qubits, these conditions do not work. Consider as an example the GHZ-type state of the form $|\Psi\rangle=x|000\rangle+y|111\rangle$, $x^{2}+y^{2}=1$. It is seen that 3-tangle (B.1) $\tau(\Psi)=4 x^{2} y^{2}=4 x^{2}\left(1-x^{2}\right)$, so 
that the state is entangled (in the three-part sector) for all $x \in(0,1)$. The reduced two-qubit density matrix for any pair of qubits has the form

$$
\rho_{R}=x^{2}|00\rangle\left\langle 00\left|+\left(1-x^{2}\right)\right| 11\right\rangle\langle 11|
$$

with the corresponding von Neumann entropy

$$
H\left(\rho_{R}\right)=-x^{2} \log x^{2}-\left(1-x^{2}\right) \log \left(1-x^{2}\right) .
$$

Subsequent reduction of $\rho_{R}$ to the single-qubit state

$$
\rho_{R R}=x^{2}|0\rangle\left\langle 0\left|+\left(1-x^{2}\right)\right| 1\right\rangle\langle 1|
$$

obviously leads to the same von Neumann entropy $H\left(\rho_{R}\right)$ as $\rho_{R}$, although there is no two-qubit entanglement in the state. Similar behavior, showing unfitness of the reduced state entropy as a general measure of entanglement, is manifested by the $W$ and $\mathrm{Bi}$ states of three qubits as well.

\section{APPENDIX C: MIXED STATE ENTANGLEMENT}

Amount of entanglement carried by a mixed single spin qutrit state can be calculated in the same way as for two qubits through the use of Wootters' concurrence. ${ }^{(36)}$ Namely

$$
\mu(\rho)=\max \left(0, \lambda_{1}-\lambda_{2}-\lambda_{3}\right)
$$

where $\lambda_{i}$ are the square roots of the eigenvalues of the $(3 \times 3)$ matrix $\rho F \rho^{*} F$, in decreasing order. Here the "spin-flip" transformation matrix $F$ is defined for the spin qutrit state as follows

$$
F=\left(\begin{array}{lll}
0 & 0 & -1 \\
0 & 1 & 0 \\
-1 & 0 & 0
\end{array}\right)
$$

It corresponds to the Wootters' spin-flip transformation $\sigma_{y} \otimes \sigma_{y}$ defined in the symmetric sector of the four-dimensional Hilbert space.

For example, the concurrence (C.1) of the "symmetric" Werner state

$$
\rho_{W}=\frac{x}{3}(|+1\rangle\langle+1|+| 0\rangle\langle 0|+|-1\rangle\langle-1|)+(1-x)|0\rangle\langle 0|,
$$


which represents superposition of completely mixed and completely entangled states, has the form

$$
C\left(\rho_{W}\right)= \begin{cases}(1-4 x / 3), & \text { at } 0 \leq x<3 / 4 \\ 0, & \text { at } 3 / 4 \leq x \leq 1\end{cases}
$$

Remind that in the case of conventional two-qubit Werner state ${ }^{(47)}$ the concurrence has the form

$$
C\left(\rho_{W_{4 d}}\right)= \begin{cases}(1-3 x / 2), & \text { at } 0 \leq x<2 / 3 \\ 0, & \text { at } 2 / 3 \leq x \leq 1\end{cases}
$$

Thus, entanglement of "symmetric" Werner state survives at higher admixture of completely chaotic state than that of Werner state in the whole space $\mathcal{H}_{2} \otimes \mathcal{H}_{2}$.

\section{APPENDIX D: VIOLATION OF BELL-TYPE CONDITION BY SINGLE SPIN 1}

Spin 1 state space can be represented with complexification of Euclidian space of $\mathcal{H}=\mathbb{R}^{3} \otimes \mathbb{C}$ where $\mathrm{SU}(2) \sim \mathrm{SO}(3)$ acts on $\mathcal{H}$ by rotations $\mathbb{R}^{3}$. It inherited from $\mathbb{R}^{3}$, the bilinear scalar and cross products are denoted by $(x, y)$ and $[x, y]$ respectively. Spin projection onto direction $\ell \in \mathbb{R}^{3}$ becomes

$$
S_{\ell} \psi=i[\ell, \psi]
$$

Spin projection has one real eigenstate $|0\rangle=\ell$ with eigenvalue 0 and two imaginary eigenstates $| \pm 1\rangle=\frac{1}{\sqrt{2}}(m \pm i n)$ with eigenvalues \pm 1 , where $(\ell, m, n)$ is orthonormal basis of $\mathbb{R}^{3}$ (e.g. coordinate vectors $i, j, k$ ).

Real vector $|0\rangle$ is completely entangled spin state, while complex ones are coherent, as we discussed in the paper.

Let us define operator $R_{\ell}=2 S_{\ell}^{2}-1$. While $S_{\ell}$ has three eigenvalues, $R_{\ell}$ has only two eigenvalues \pm 1 . Observe that $R_{\ell}^{2}=1$ and operators $R_{\ell}$ and $R_{m}$ commute iff $\ell \perp m$. Then they have joint probability distribution, and are simultaneously measurable observables.

Consider a cyclic quintuplet of unit vectors $\ell_{i} \in \mathbb{R}^{3}, i$ mod 5 , such that $\ell_{i} \perp \ell_{i+1}$, and call it pentagram.

Put $R_{i}:=R_{\ell_{i}}$. Then $\left[R_{i}, R_{i+1}\right]=0$ and for all possible eigenvalues $r_{i}= \pm 1$ of observable $R_{i}$

$$
\left(r_{1} r_{2}\right)\left(r_{2} r_{3}\right)\left(r_{3} r_{4}\right)\left(r_{4} r_{5}\right)\left(r_{5} r_{1}\right)=1 \text {. }
$$




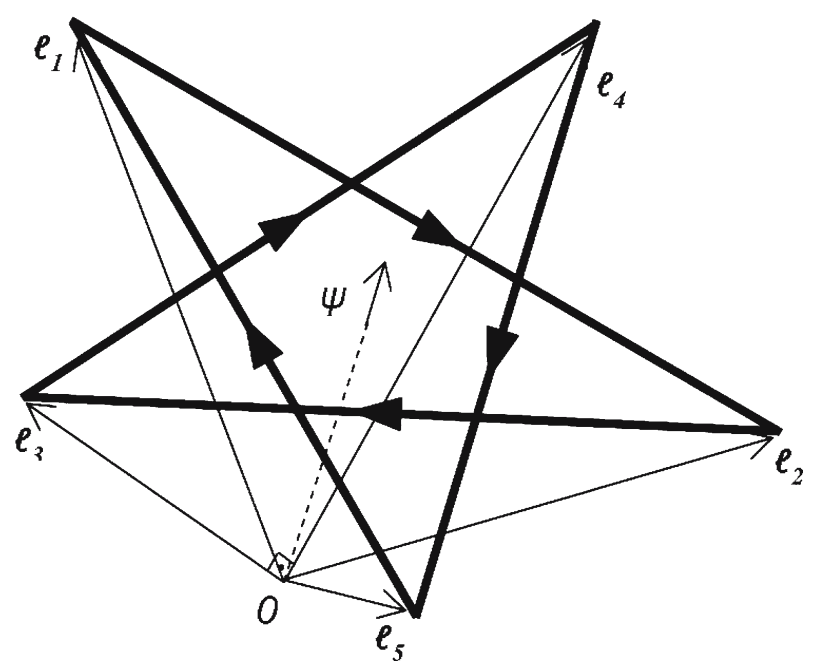

Fig. 2. Regular planar pentagram.

Among those five monomials at least one is positive. So the following inequality holds

$$
r_{1} r_{2}+r_{2} r_{3}+r_{3} r_{4}+r_{4} r_{5}+r_{5} r_{1}+3 \geq 0 .
$$

If all $R_{i}$ would have a hidden joint distribution then taking average of (D.1) one get Bell's type inequality

$$
\begin{array}{r}
\left\langle\psi\left|R_{1} R_{2}\right| \psi\right\rangle+\left\langle\psi\left|R_{2} R_{3}\right| \psi\right\rangle+\left\langle\psi\left|R_{3} R_{4}\right| \psi\right\rangle \\
+\left\langle\psi\left|R_{4} R_{5}\right| \psi\right\rangle+\left\langle\psi\left|R_{5} R_{1}\right| \psi\right\rangle+3 \geq 0
\end{array}
$$

for testing classical realism.

Using the identity $R_{i}=1-2\left|\ell_{i}\right\rangle\left\langle\ell_{i}\right|$ one can recast it into geometrical form

$$
\sum_{i \bmod 5}\left|\left\langle\ell_{i}, \psi\right\rangle\right|^{2} \leq 2 \Longleftrightarrow \sum_{i \bmod 5} \cos ^{2} \alpha_{i} \leq 2, \quad \alpha_{i}=\widehat{\ell_{i} \psi} .
$$

Completely entangled spin states easily violate this inequality. Say for regular pentagram (Fig. 2) and $\psi \in \mathbb{R}^{3}$ directed along its axis of symmetry one gets

$$
\sum_{i \bmod 5} \cos ^{2} \alpha_{i}=\frac{5 \cos \pi / 5}{1+\cos \pi / 5} \approx 2.236>2 .
$$


It turns out that every non-coherent spin state violates inequality (D.3) for an appropriate pentagram, and the coherent states pass this test for any pentagram, see the Ref. 14 for details.

\section{REFERENCES}

1. L. Marcikic, H. de Riedmatten, W. Tittel, and N. Gisin, Nature (London) 42, 509 (2003); A. Poppe, A. Fedrizzi, R. Ursin, H. R. Böhm, T. Lörunser, O. Maurhardt, M. Peev, M. Suda, C. Kurtsiefer, H. Weinfurter, T. Jennewein, and A. Zeilinger, Optics Express 12, 3865 (2004); J. Ouelette, Ind. Phys. 10, 22 (2004).

2. R. C. S. Spreeuw, Found. Phys. 28, 361 (1998); A. Zeilinger, Found. Phys. 29, 631 (1999); A. Cabello, Found. Phys. 35, 1927 (2005); Č. Brukner et al., Found. Phys. 35, 1909 (2005); D. P. DiVincenzo et al., Found. Phys. 33, 1629 (2003); K. Horodecki et al., Found. Phys. 35, 2027 (2005); D. I. Fivel, Found. Phys. 29, 561 (1999); H. A. Carteret et al., Found. Phys. 29, 527 (1999); D. Bruß et al., Found. Phys. 33, 1617 (2004); P. Schust et al., Found. Phys. 34, 99 (2004); G. Auletta, Found. Phys. 35, 787 (2005); J. Bub, Found. Phys. 35, 541 (2005); T. Krüger, Found. Phys. 30, 1869 (2000); F. Hulpke et al., Found. Phys. 36, 477 (2006); P. Holland, Found. Phys. 35, 177 (2005).

3. A. Einstein, B. Podolsky, and N. Rosen, Phys. Rev. 47, 777 (1935).

4. M. A. Nielsen and I. L. Chuang, Quantum Computations and Quantum Information (Cambridge University Press, New York, 2000).

5. J. S. Bell, Rev. Mod. Phys. 38, 447 (1966).

6. A. A. Klyachko and A. S. Shumovsky, J. Phys. Conf. Series 36, 87 (2006), E-print quant-ph/0512213.

7. A. A. Klyachko and A.S. Shumovsky, J. Opt. B: Quant. Semiclas. Opt. 5, S322 (2003).

8. S. J. van Enk, Phys. Rev. A 67, 022303 (2003); Phys. Rev. A 72, 064306 (2005).

9. H. Barnum, E. Knill, G. Ortiz, R. Somma, and L. Viola, Phys. Rev. Lett. 92, 107902 (2004).

10. M. A. Can, A. A. Klyachko, and A. S. Shumovsky, J. Opt. B: Quant. Semiclass. Opt. 7, L1 (2005).

11. A. A. Klyachko, E-print quant-ph/0206012.

12. H. Barnum, E. Knill, G. Ortiz, and L. Viola, Phys. Rev. A 68, 032308 (2003).

13. A. A. Klyachko, J. Phys.: Conf. Series 36, 87 (2006).

14. A. A. Klyachko, in Physics and Theoretical Computer Science From Numbers and Languages to (Quantum) Cryptography Volume 7, J.-P. Gazeau, J. Ned́cetril, and B. Rovan, eds. NATO Security through Science Series: Information and Communication Security (IOS Press, Amsterdam, 2007).

15. A. Peres, Quantum Theory: Concepts and Methods (Kluwer Academic, Dordrecht, 1995).

16. D. Bruß, J. Math. Phys. 43, 4237 (2002).

17. A. Miyake, Phys. Rev. A 67, 012108 (2003).

18. M. A. Can, A. A. Klyachko, and A. S. Shumovsky, Phys. Rev. A 66, 022111 (2002).

19. O. Gühne, P. Hyllus, D. Bruß, A. Ekert, M. Lewenstein, C. Macchiavello, and A. Sanpera A, Phys. Rev. A 66, 062305 (2002); H. F. Hofmann and S. Takeuchi, Phys. Rev. A 68, 032103 (2003); B.-Q. Jin and V. E. Korepin, Phys. Rev. A 69, 062314 (2004).

20. J. M. G. Sancho, S. F. Huelga, Phys. Rev. A 61, 042303 (2000). 
21. M. Hayashi, B.-S. Shi, A. Tomita, K. Matsumoto, Y. Tsuda, and Y.-K. Jiang, E-print quant-ph/0603254.

22. A. A. Klyachko and A. S. Shumovsky, J. Opt. B: Quant. Semicla. Opt. 6, S29 (2004).

23. R. Hermann, Lie Groups for Physicists (Benjamin, New York, 1966).

24. W. Dür, G. Vidal, and J. I. Cirac, Phys. Rev. A 62, 062314 (2000).

25. F. Verstraete, J. Dehaene, and B. De Moor, Phys. Rev. A 68, 012103 (2003).

26. A. V. Burlakov, M. V. Chechova, O. A. Karabutova, D. N. Klyshko, and S. P. Kulik, Phys. Rev. A 60, R4209 (1999); H. Bechman-Pasquinucci and A. Peres, Phys. Rev. Lett. 85, 3313 (2000); D. Bruß and C. Macchiavello, Phys. Rev. Lett. 88, 127901 (2002).

27. C. M. Caves and G. J. Milburn, Opt. Commun. 179, 439 (2000).

28. L. Hardy, Phys. Rev. Lett. 73, 2279 (1994); C. C. Gerry, Phys. Rev. A 53, 4583 (1996); P. G. Kwiat and R. J. Hughes, Science, 289, 1431 (2000); A. Beige, B.-G. Englert, C. Kurtsifer, and H. Weinfurter, J. Phys. A: Math. Gen. 35, L407 (2002); Y. H. Kim, Phys. Rev. A 67, 040301 (2003).

29. J. M. Radcliffe, J. Phys. A: Math. General 4, 313 (1971): F. T. Arecchi, E. Courtens, R. Gilmore, and H. Thomas, Phys. Rev. A 6, 2211 (1972).

30. A. M. Perelomov, Generalized Coherent States and Their Applications (Springer, Berlin, 1986); J. Peřina, Quantum Statistics of Linear and Nonlinear Optical Phenomena (Kluwer Academic, Dordrecht, 1991).

31. J. Hald, J. L. Sørensen, C. Schori, and E. S. Polzik, Phys. Rev. A 83, 1319 (1999); A. Sørensen, L. M. Duan, J. I. Cirac, and P. Zoller, Nature (London) 409, 63 (2001); U. V. Poulsen and K. Mølmer, Phys. Rev. A 64, 013616 (2001); A. Sørensen and K. Mølmer, Phys. Rev. Lett. 86, 4431 (2001); L. K. Thomsen, S. Mancini, and H. M. Wiseman, Phys. Rev. A 65, 061801 (2002); J. K. Stokton, J. M. Geremia, A. C. Doherty, and H. Mabuchi, Phys. Rev. A 67, 022112 (2003); D. W. Berry and B. C. Sanders, J. Phys. A 38, L 205 (2005).

32. P. Rungta, V. Bužek, C. M. Caves, M. Hillery, and G. J. Milburn, Phys. Rev. A, 64, 042315 (2001).

33. E. P. Wigner and M. M. Yanase, Proc. Nat. Acad. Sci. USA 19, 910 (1963).

34. H. Araki and M. M. Yanase, Phys. Rev. 120, 622 (1960); F. J. Dyson and A. Leonard, J. Math. Phys. 8, 423 (1967); F. J. Dyson, J. Math. Phys. 8, 1538 (1967); E. H. Lieb and M. B. Ruskai, Phys. Rev. Lett. 30, 434 (1973); A. Uhlman, Comm. Math. Phys. 54, 21 (1977); M. Ozawa, Phys. Rev. Lett. 88, 050402 (2002); J. Řeháček and Z. Hradil, Phys. Rev. Lett. 90127904 (2003); S.-L. Luo, Phys. Rev. Lett. 91, 180403 (2003); P. Gibilisco and T. Isola, J. Math. Phys. 44, 3752 (2003); S.-L. Luo and Q. Zhang, Phys. Rev. A 69, 032106 (2004).

35. A. A. Klyachko, B. Öztop, and A. S. Shumovsky, Appl. Phys. Lett. 88, 124102 (2006).

36. Concurrence as a measure of two-qubit entanglement has been introduced by W. K. Wooters, Phys. Rev. Lett. 80, 2245 (1998). For its generalization on an arbitrary bipartite state, see: P. Rungta, V. Bužek, C.M. Caves, M. Hillery, and G. J. Milburn, Phys. Rev. A, 64, 042315 (2001); F. Minnert, M. Kuś, and A. Buchleitner, Phys. Rev. Lett. 92, 167902 (2004).

37. M. Kitagawa and M. Ueda, Phys. Rev. A 47, 5138 (1993).

38. Ö. Çakır, A. A. Klyachko, and A. S. Shumovsky, Phys. Rev. A 71, 034303 (2005).

39. A. V. Burlakov, M. V. Chechova, O. A. Karabutova, D. N. Klyshko, and S. P. Kulik, Phys. Rev. A 60, R4209 (1999); M. B. Nasr, A. F. Abouraddy, M. C. Booth, B. E. A. Saleh, A. V. Sergienko, M. C. Teich, U. Kempe, amd R. Wollescheensky, Phys. Rev. A 65, 023816 (2002); G. DiGiuseppe, M. Atature, M. Shaw, A. V. Sergienko, B. E. A. Saleh, M. C. Teich, A. J. Miller, S. W. Nam, and J. M. Martinis, Phys. Rev. A 68, 063817 (2003); Y. I. Bogdanov, M. V. Chechova, S. P. Kulik, 
G. A. Maslennikov, A. A. Zhukov, C. H. Oh, and M. K. Tey Phys. Rev. Lett. 93, 230503 (2004); M. V. Chechova, L. A. Krivitsky, S. P. Kulik, and G. A. Maslennikov, Phys. Rev. A 70, 053801 (2004).

40. P. Wölfe, The Superfluid Phases of Helium 3 (Taylor \& Francis, London, 1990).

41. J. M. Blatt and V. F. Weisskopf, Theoretical Nuclear Physics (Wiley, New York, 1952); R. J. Blin-Stoyl, Nuclear and Particle Physics (Chapman \& Hall, London, 1991).

42. S. Hamieh, J. Phys.: Math. Gen. 37, L59 (2004).

43. N. Cottingham and D. A. Greenwood, An Introduction to Standard Model of Elementary Particle Physics (Cambridge University Press, New York, 1998).

44. J. von Neumann, Mathematical Foundations of Quantum Mechanics (Princeton University Press, Princeton, 1955).

45. V. Coffman, J. Kundu, and N. K. Wootters, Phys. Rev. A, 61, 052306 (2000).

46. R. Horodecki, P. Horodecki, and M. Horodecki, Phys. Lett. A 210, 377 (1996).

47. R. F. Werner, Phys. Rev. A 40, 4277 (1989). 